Diseases Unit, Central Adelaide Local Health Network, Adelaide, SA, Australia

Arthrographis kalrae is a saprophytic fungus with initially yeastlike colonies, eventually developing hyphae and distinctive cylindrical arthroconidia. A. kalrae is rarely associated with human infection. This study reports the first case of prosthetic joint infection.

A 75-year-old male with a background of type 2 diabetes, obesity and total knee replacement underwent debridement and polyliner exchange for chronic methicillin-resistant Staphylococcus aureus (MRSA) prosthetic joint infection. He was treated with vancomycin for 6 weeks and oral trimethoprim/sulfamethoxazole (SXT) for 18 months. Although the wound remained intact, knee inflammation progressed after cessation of SXT. First stage revision revealed loose femoral and tibial components, tibial and patellar bone defects, and turbid synovial fluid. Histology demonstrated chronic inflammation and small branching hyphae but no neutrophils. MRSA was not isolated however cultures from multiple specimens grew a fungus. The isolate was referred to the National Mycology Reference Centre (NMRC) for confirmation and susceptibility testing, where morphological features were consistent with Arthrographis spp. A. kalrae was confirmed using the Bruker Biotyper MALDI-ToF MS (Score 2.28).

Liposomal amphotericin for 2 weeks followed by voriconazole and terbinafine were given in conjunction with repeated debridement and voriconazole-infused cement. A review of the literature and the implications of prosthetic joint associated fungal infection will be discussed.

\section{COMPARATIVE EVALUATION AND LONGEVITY OF NEUTRALISING AND BINDING ANTIBODIES FOR SARS-COV-2}

Natalie Davidson ${ }^{1}$, Jenny Evans ${ }^{2}$, Jenny Robson

${ }^{1}$ Department of Microbiology, Sullivan and Nicolaides Pathology, Brisbane, Qld, Australia; and ${ }^{2}$ Department of Immunology/Serology, Sullivan and Nicolaides Pathology, Brisbane, Qld, Australia

There are many unanswered questions regarding serological testing for SARS-CoV-2 including the longevity of the neutralising antibody response and correlation with binding antibodies and subsequent protection. This study evaluated the durability of neutralising antibody response over a 12-month period using the cPass $^{\text {TM }}$ SARS-CoV-2 sVNT and correlation with binding antibodies using both the LIAISON® SARS-CoV-2 S1/S2 IgG and Abbott Architect SARS-CoV-2 IgG. 214 samples were evaluated from 81 SARS-CoV-2 NAAT confirmed positive patients collected from March 2020-March 2021 at various time intervals post symptom onset. 120 samples from a reference non-infected population were used for specificity analysis. The $\mathrm{cPass}^{\mathrm{TM}}$ SARS-CoV-2 sVNT had the highest overall sensitivity $90.3 \%$ [CI 84.9, 93.1]. Specificity was comparable to both binding antibody assays, 98.33 [CI 95.4, 99.96]. Sensitivity peaked at 3-6 months post symptom onset at 100\% [CI 91.4, 100.0] and persisted up to $9-12$ months post infection with sensitivity of $78.8 \%$ [CI 62.3, 89.3]. S1/S2 binding antibodies demonstrated satisfactory correlation with RBD neutralising antibodies (Spearman coefficient 0.8937 [CI 0.86,0.92]); nucleocapsid antibodies, however, waned markedly 6 months following infection. There was an association with clinical severity and degree of neutralising antibody response $(p=0.0025)$. No association between age or gender was demonstrated in this study. These results support both the use of the cPass ${ }^{\mathrm{TM}}$ SARS-CoV-2 sVNT to assess neutralising antibodies and the possible use S1/ S2 binding antibodies as a surrogate for neutralising antibodies in diagnostic laboratories; as well as provides information on durability of neutralising antibody response and kinetics over time as the COVID-19 pandemic unfolds.

\section{LABORATORY SAFETY: HANDLING $B$. PSEUDOMALLEI ISOLATES WITHOUT A BIOSAFETY CABINET}

$\underline{\text { Ian Gassiep }}^{1,2}$, Michelle J. Bauer ${ }^{1}$, Patrick N. A. Harris ${ }^{1,3}$, Mark D. Chatfield ${ }^{1}$, Robert Norton ${ }^{4}$

${ }^{1}$ University of Queensland Centre for Clinical Research, Royal Brisbane and Woman's Hospital, Herston, Qld, Australia; ${ }^{2}$ Department of Infectious Diseases, Mater Hospital Brisbane, South Brisbane, Qld, Australia; ${ }^{3}$ Pathology Queensland, Royal Brisbane \& Women's Hospital, Herston, Qld, Australia; and ${ }^{4}$ Pathology Queensland, Townsville University Hospital,

Townsville, Qld, Australia

Background: $B$. pseudomalle $i$ is a tier 1 select agent that is associated with laboratory-acquired melioidosis, with international guidelines recommending isolate handling within a class II biosafety cabinet (BSC) in a Biosafety Level (BSL) 3 facility. In low-resource settings this may not be practical, and therefore the authors aimed to assess the risk of laboratory-acquired melioidosis during routine work.

Methods: A questionnaire combined with two serological assays were conducted to determine prior exposure to the organism. Additionally, we performed controlled environmental air sampling during 78 laboratory handling events including plate opening, oxidase testing, and McFarland suspension creation.

Results: Of 30 laboratory scientists to handle $B$. pseudomallei on 1,267 occasions outside a biosafety cabinet no infections were documented and all participants remained seronegative. None of the experiments demonstrated aerosolisation of the organism.

Conclusion: This study suggests the risk of laboratory-acquired melioidosis is likely to be low. However, individual laboratories will need to undertake a risk assessment including melioidosis endemicity, availability of resources for containment, the nature of routine handling to be undertaken, and the presence of predisposing risk factors for infection in the staff concerned. Further research is required to better understand on the risk of infection in the microbiology laboratory.

Contribution: IG conceived of the idea, obtained funding, obtained ethics approval, performed bioaerosol experiments and contributed to each section of the manuscript.

Publication statement: This research has been published in the Journal of Clinical Microbiology.

\section{NEONATAL CANDIDAEMIA; SIX-YEAR EXPERIENCE FROM A TERTIARY CARE CENTRE IN SRI LANKA}

G. P. S. Gunaratna ${ }^{1}$, M. G. T. D. Wijewardhane ${ }^{1}$,

A. Sutharson ${ }^{1}$, U. A. G. H. Wickramasuriya ${ }^{1}$,

Y. C. Waniganayake ${ }^{1}$, K. K. M. K. Premaratne ${ }^{1}$,

J. Galappaththi ${ }^{1}$, D. Somasiri ${ }^{1}$, S. M. P. Karunaratne ${ }^{1}$, 
G. S. B. Wijethunga ${ }^{1}$, S. D. Amarasinghe ${ }^{1}$, T. M. R. Perera ${ }^{1,2}$, P. I. Jayasekera ${ }^{3}$, R. Sathanandaraja ${ }^{1}$, N. S. Chandrasiri ${ }^{1}$ ${ }^{1}$ Colombo South Teaching Hospital, Kalubowila, Sri Lanka;

${ }^{2}$ University of Sri Jayewardenepura, Nugegoda, Sri Lanka; and

${ }^{3}$ Medical Research Institute, Colombo 08, Sri Lanka

Aim: Candida species causing neonatal candidaemia show a wide geographical variation and are usually healthcare acquired. Awareness of prevalent Candida species and knowledge of possible risk factors allows timely commencement of antifungal prophylaxis, directs appropriate empirical antifungal therapy and helps to prevent occurrence. This study aims to evaluate the Candida species distribution and the impact on preventive measures.

Method: This was carried out as a prospective descriptive study in a Special Care Baby Unit (with a capacity of 16 cots with ventilation facilities for 8 newborns) attached to a tertiary care hospital in Colombo, Sri Lanka from January 2015 to December 2020. Identification and antifungal sensitivities were done according to standard protocols. Impact of preventive measures implemented to prevent nosocomial transmission of Candida species was reassessed.

Result: A total of 27 candidaemia episodes (C. parapsilosis $61.54 \%$, C. albicans $15.38 \%$, C. guilliermondii $15.38 \%$ and $7.7 \%$ non-albicans Candida) were detected during the study period. The overall incidence was 1.04 per 100 SCBU admissions. A drop in antibiotic consumption was noted with antibiotic stewardship implementation.

Discussion: Neonatal candidaemia was predominantly caused by non-albicans Candida species. Implementation of antibiotic stewardship programme and strengthening of infection control activities markedly reduced the incidence of neonatal candidaemia.

\section{THE UTILITY OF THE SYPHILIS ENZYME IMMUNOASSAY IGM IN THE DIAGNOSIS OF CONGENITAL SYPHILIS}

Stephanie Hendry $^{1}$, Madeleine Kelly ${ }^{2}$, Robert Norton ${ }^{1}$ ${ }^{1}$ Pathology Queensland, Townsville Hospital, Townsville, Qld, Australia; and ${ }^{2}$ School of Medicine, James Cook University, Townsville, Qld, Australia

Background: Congenital syphilis has increasing incidence in Australia, with significant sequelae. Effective screening tests are available during pregnancy; however, they rely on adequate antenatal care. Congenital syphilis can be a challenging diagnosis to make, with a variety of clinical and laboratory factors to consider. The syphilis IgM routinely ordered as part of the diagnostic process.

Aim: To assess the utility of the syphilis EIA IgM test in the diagnosis and treatment of congenital syphilis.

Methods: Laboratory and clinical records of mothers and newborns who were syphilis EIA IgM reactive were reviewed over a ten-year period.

Results: There were 11 syphilis IgM reactive results from 371 tests performed $(3 \%)$. Nine of these cases were reviewed in detail. The IgM result was never a key factor in establishing a diagnosis or instigating treatment in an infant.

Discussion: Use of maternal serology and treatment history are better tools to identify those neonates at risk of congenital syphilis, rather than the EIA syphilis IgM result. In cases when antenatal maternal serology is unavailable, PCR can provide valuable a timely insight in at risk neonates. This study highlights the low value of the syphilis IgM in the diagnostic management of congenital syphilis.

\section{SPINAL TOXOPLASMOSIS IN A YOUNG MAN WITH UNDIAGNOSED HIV INFECTION}

Annaleise R. Howard-Jones ${ }^{1,2}$, Zoe Jennings ${ }^{2,3}$, Stuart Adams $^{1,2}$, Harsha Samarasekara ${ }^{1,2}$

${ }^{1}$ NSW Health Pathology (Nepean), Nepean Hospital, Kingswood, NSW, Australia; ${ }^{2}$ Faculty of Medicine \& Health, University of Sydney, Camperdown, NSW, Australia; and ${ }^{3}$ Department of Microbiology \& Infectious Diseases, Nepean Hospital, Kingswood, NSW, Australia

Spinal cord lesions are potentially devastating conditions leading to reduced mobility, loss of functional independence, and even ventilator dependence, dictated by the level of injury. Tissue diagnosis is paramount to differentiate between primary brain tumours such as ependymomas and glioblastomas, infarction, infection and demyelination.

We present the case of a 30-year-old gentleman with subacute neck pain, nausea and evolving right upper limb weakness and paraesthesiae. MRI-spine demonstrated an intramedullary cervical spine mass with associated oedema, thought to represent an ependymoma. Open tissue biopsy revealed numerous bradyzoites on haematoxylin \& eosin staining, subsequently confirmed by directed polymerase chain reaction testing as Toxoplasma gondii. Detailed immunological work-up led to a diagnosis of HIV infection, with detection of HIV antibodies and HIV-1 p24 antigen, an HIV-1 viraemia ( $\log 6.33$ copies $/ \mathrm{mL})$ and low $\mathrm{CD} 4$ count $(16$ cells $/ \mathrm{mL})$.

Toxoplasmosis is the most common central nervous system infection in HIV patients not receiving appropriate prophylaxis. ${ }^{1}$ It may occur in up to $30 \%$ of such patients who are seropositive for Toxoplasma gondii, ${ }^{2}$ typically manifesting when CD4+ counts fall below 100 cells $/ \mathrm{mm}^{3}{ }^{3}$. This case highlights the importance of close collaboration between histopathology, microbiology and infectious diseases specialists in diagnosis of a spinal cord infection with associated HIV infection.

References

1. Porter SB, Sande MA. Toxoplasmosis of the central nervous system in the acquired immunodeficiency syndrome. $\mathrm{N} \mathrm{Engl} \mathrm{J}$ Med 1992; 327: 1643-8.

2. Grant IH, Gold JW, Rosenblum M, et al. Toxoplasma gondii serology in HIV-infected patients: the development of central nervous system toxoplasmosis in AIDS. AIDS 1990; 4: 519-21.

3. Kaplan JE, Benson C, Holmes KK, et al. Guidelines for prevention and treatment of opportunistic infections in HIVinfected adults and adolescents. MMWR Recomm Rep 2009; 58: $1-207$.

\section{EXPRESSION OF GPI-ANCHORED SARS-COV-2 RECEPTOR BINDING DOMAIN IN MAMMALIAN CELLS FOR SEROLOGICAL USE}

M. L. Kernich ${ }^{1,2}$, P. J. Adamson ${ }^{2}$, J. J. Wang ${ }^{3}$, L. P. Kris ${ }^{3}$, B. L. Armour ${ }^{3}$, D. Beroukas ${ }^{2}$, G. Best ${ }^{3}$, J. M. Carr ${ }^{3}$, T. P. Gordon ${ }^{2,3,4}$, D. L. Gordon ${ }^{1,2,3}$

${ }^{1}$ Microbiology and Infectious Diseases, Flinders Medical Centre, SA, Australia; ${ }^{2}$ SA Pathology, SA, Australia; ${ }^{3}$ College 